\title{
An Algorithm for Solving a Certain Class of Diophantine Equations. I
}

\author{
By David Lee Hilliker
}

\begin{abstract}
A class of Diophantine equations is defined and an algorithm for solving each equation in this class is developed. The methods consist of techniques for the computation of an upper bound for the absolute value of each solution. The computability of these bounds is guaranteed. Typically, these bounds are well within the range of computer programming and so they constitute a practical method for computing all solutions to the Diophantine equation in question. As a first application, a bound for a cubic equation is computed. As a second application, a set of quartic equations is studied. Methods are developed for deriving various sets of conditions on the coefficients in such equations under which a bound exists and can be computed.
\end{abstract}

1. Introduction. Traditionally, the term Diophantine equation usually refers to an equation, $F\left(x_{1}, x_{2}, \ldots, x_{n}\right)=0$, in $n$ integer variables, $x_{1}, x_{2}, \ldots, x_{n}$, where $F$ is a polynomial, with integer coefficients, in $n$ variables. Here, we use the term in a more general sense, where the equation is not required to be a polynomial equation.

Diophantine equations can, and often do, have only finitely many solutions. A well-studied illustration of this, which encompasses a substantial portion of the theory of Diophantine equations, is furnished by Mordell's equation, $y^{2}+k=x^{3}$. This Diophantine equation is known to have only finitely many solutions, in the case that $k$ is a given nonzero integer. For example, the Diophantine equation $y^{2}-17=$ $x^{3}$ was completely solved by Nagell [12], in 1930, by employing algebraic number theory techniques. He calculated the solutions to be:

$$
\begin{gathered}
x=-2, \quad y= \pm 3 ; \quad x=-1, \quad y= \pm 4 ; \quad x=2, \quad y= \pm 5 ; \\
x=4, \quad y= \pm 9 ; \quad x=8, \quad y= \pm 23 ; \quad x=43, \quad y= \pm 282 \\
x=52, \quad y= \pm 375 ; \quad \text { and } \quad x=5234, \quad y= \pm 378661 .
\end{gathered}
$$

For more on Mordell's equation, see Baker [1], Hemer [2], Hilliker and Steiner [4], London and Finkelstein [7], and Mordell [10], [11]. (Note: R. Finkelstein is now known as R. Steiner.)

If the Diophantine equation, $F\left(x_{1}, x_{2}, \ldots, x_{n}\right)=0$, does have only finitely many solutions, then there is, in theory, a bound of the form $\left|x_{i}\right| \leqslant B$ for each solution $x_{i}$, $i=1,2, \ldots, n$. Here, the constant $B$ depends on the function $F$ but not on the variables $x_{1}, x_{2}, \ldots, x_{n}$ or the index $i$. If one had a procedure for actually computing $B$, for a given Diophantine equation in a certain class, and if the resulting computed value were of a reasonable order of magnitude, then such a procedure would constitute an algorithm for computing all solutions to the Diophantine equation.

Received May 6, 1980; revised December 10, 1980 and June 15, 1981.

1980 Mathematics Subject Classification. Primary 10B10, 10B15, $10 \mathrm{~B} 25$.

Key words and phrases. Computability, Diophantine equation. 
In Part I of this paper we shall define a certain class of Diophantine equations and develop an algorithm for solving each equation in this class. Our methods, which are not restricted to equations in two variables, consist of the computation of bounds. The resulting bounds will, in typical cases, lend themselves to computer programming, and, in fact, will ofter lend themselves to actual hand calculation. The methods are introduced in Section 2, Part I, of this paper. In Section 3, a specially chosen example, to wit one that involves only a minimal amount of ceremony, is given. In Section 6, we shall study a specially chosen quartic equation, one with unspecified coefficients. We shall illustrate there, techniques for deriving various sets of conditions on the coefficients of such equations under which a bound exists and can be computed.

In Part II of this paper (Hilliker [3]) we shall turn to more realistic examples. There, as a further illustration of the techniques, we shall study the general quartic polynomial Diophantine equation with integer coefficients, in two variables. We shall prove there, under certain hypotheses, that the general quartic equation has only finitely many solutions and that a bound can be computed. This will amount to proving anew, by my methods, the quartic case of a theorem established by Runge [13], in 1887. This will involve extending the methods of Part I of this paper and coupling the extended methods with some numerical techniques in the classical theory of algebraic functions. See Section 9 of Part I of this paper for a precise statement of the hypotheses for the general quartic equation.

For more on Runge's Theorem, see, in addition to Hilliker [3], and Runge [13], the works of Hilliker and Straus [5], [6], Maillet [8], [9], Mordell [11], Schinzel [14], and Skolem [15], [16].

It is not automatically the case that the general quartic equation possesses a bound. Indeed, the Diophantine equation $y^{2}=\left(a x^{2}+b x+c\right)^{2}$, where $a, b$, and $c$ are given integers, has infinitely many solutions, and hence, no possible bound. A less obvious special case of the general quartic equation that has infinitely many solutions would be given by

$$
\left(x^{2}-r y^{2}-1\right)\left(a x^{2}+b x y+c y^{2}+d x+e y+f\right)=0,
$$

where $a, b, c, d, e, f$, and $r$ are given integers with $r$ positive and not equal to a square. This is due to the fact that Pell's equation, $x^{2}-r y^{2}=1$, would then have infinitely many solutions. These examples are both reducible. An irreducible quartic Diophantine equation can also have infinitely many solutions. An obvious example would be $y^{4}-x^{3}=0$.

We shall employ two functions of a real variable $w$. The first is the greatest integer function, denoted by $[w]$, and defined to be the largest integer less than or equal to $w$. The second is the fractional part function, denoted by $\{w\}$, and defined by

$$
w=[w]+\{w\}
$$

so that $0 \leqslant\{w\}<1$.

2. The Algorithm. Suppose that $n$ is a given positive integer, that, for each $i=1,2, \ldots, n, I_{i}$ is a set of integers, and that $x_{i}$ is an integer variable in $I_{i}$. Let $D=I_{1} \times I_{2} \times \cdots \times I_{n}$ be the Cartesian product consisting of all $\left(x_{1}, x_{2}, \ldots, x_{n}\right)$. We wish to consider Diophantine equations of the form $f\left(x_{1}, x_{2}, \ldots, x_{n}\right)=k$, where 
$k$ is a real constant and $f$ is a real-valued function of $n$ variables, defined for all $\left(x_{1}, x_{2}, \ldots, x_{n}\right)$ in the domain $D$. We introduce an integer $m$ in the range $1 \leqslant m \leqslant n$ to accommodate the situation of a Diophantine equation that requires one set of techniques to solve for the first $m$ variables, $x_{1}, x_{2}, \ldots, x_{m}$, and that requires a modified set of techniques to solve for the possible $n-m$ remaining variables, $x_{m+1}, x_{m+2}, \ldots, x_{n}$. For example, the Diophantine equation could have only finitely many solutions in the first $m$ variables and infinitely many in each of the $n-m$ remaining variables.

We begin by defining a class $\Delta$, after Diophantus, of Diophantine equations of the above type, where there are only finitely many solutions in the first $m$ variables for all choices of the possible $n-m$ remaining variables. We next define a subclass $\Delta_{1}$ of $\Delta$ consisting of all such Diophantine equations where there are only finitely many solutions in the first $m$ variables for all choices of the possible $n-m$ remaining variables, and for all choices of the real constant $k$ in any interval of finite length.

We realize then that there is, in theory, a bound of the form $\left|x_{i}\right| \leqslant B(k)$, for each Diophantine equation in each of the above defined classes $\Delta$ and $\Delta_{1}$, for each $i \leqslant m$, where $B$ depends on $k$ and the function $f$, but not on the variables $x_{1}, x_{2}, \ldots, x_{n}$, or the index $i$.

We also realize that there are Diophantine equations in $\Delta$ that are not in $\Delta_{1}$. An example of this would be $1 / x+1 / y=k$. For $k=2$, this equation has only the solution $(1,1)$; but if $k$ varies over the interval [1,2], in the infinite sequence $k=2$, $3 / 2,4 / 3,5 / 4, \ldots$, there are infinitely many solutions $(1,1),(1,2),(1,3),(1,4), \ldots$.

Our objective will be to characterize the classes $\Delta$ and $\Delta_{1}$ in terms of the existence of a certain quantity, $q=q\left(x_{1}, x_{2}, \ldots, x_{n}\right)$, defined for all $\left(x_{1}, x_{2}, \ldots, x_{n}\right)$ in $D$. Then, based upon this characterization, we shall define another class $\Delta_{2}$, a subclass of $\Delta_{1}$, which will turn out to contain only Diophantine equations that possess a computable bound. By proceeding in this manner, we will arrive at the point of view that: the computation of the solutions to a Diophantine equation rests upon the computation of a certain function $q=q\left(x_{1}, x_{2}, \ldots, x_{n}\right)$ with certain specified properties. We shall associate with $q$ a second function $\omega=\omega\left(x_{1}, x_{2}, \ldots, x_{n}\right)$, defined for all $\left(x_{1}, x_{2}, \ldots, x_{n}\right)$ in $D$ by

$$
\omega=\left\{\frac{f\left(x_{1}, x_{2}, \ldots, x_{n}\right)}{q\left(x_{1}, x_{2}, \ldots, x_{n}\right)}\right\} .
$$

We now state our algorithm in the form of three theorems.

THEOREM 1. A given Diophantine equation is in the class $\Delta$ if and only if there is a real-valued function $q$ of $n$ variables, defined for all $\left(x_{1}, x_{2}, \ldots, x_{n}\right)$ in $D$, with $q\left(x_{1}, x_{2}, \ldots, x_{n}\right) \neq 0$ for all $\left(x_{1}, x_{2}, \ldots, x_{n}\right)$ in $D$, and that possesses the following three properties, for all solutions $\left(x_{1}, x_{2}, \ldots, x_{n}\right)$ in $D$ to the Diophantine equation. First, it is required that

(i) $k q\left(x_{1}, x_{2}, \ldots, x_{n}\right)>0$, if $k \neq 0$. Secondly, it is required that, for each $i \leqslant m$,

(ii) $\lim \left|q\left(x_{1}, x_{2}, \ldots, x_{n}\right)\right|=\infty$, uniformly in $x_{1}, x_{2}, \ldots, x_{i-1}, x_{i+1}, \ldots, x_{n}$, as $\left|x_{i}\right|$ approaches infinity. Finally, it is required that $\omega$ satisfy, for each $i \leqslant m$, 
(iii) $\omega\left|q\left(x_{1}, x_{2}, \ldots, x_{n}\right)\right| \neq|k|$, if $\left|x_{i}\right|>x_{0}$, for some constant $x_{0}$, independent of $x_{1}, x_{2}, \ldots, x_{n}$ and $i$.

Theorem 2. A given Diophantine equation is in the class $\Delta_{1}$ if and only if there is such a function $q$ which satisfies, for all solutions $\left(x_{1}, x_{2}, \ldots, x_{n}\right)$ in $D$ to the Diophantine equation, the condition (i), but where (ii) and (iii) are replaced by the stronger set of conditions that, for each $i \leqslant m$,

(iv) $\lim \omega\left|q\left(x_{1}, x_{2}, \ldots, x_{n}\right)\right|=\infty$, uniformly in $x_{1}, x_{2}, \ldots, x_{i-1}, x_{i+1}, \ldots, x_{n}$, as $\left|x_{i}\right|$ approaches infinity.

We note that the conditons (i), (ii), (iii), and (iv) are required to hold only for solutions $\left(x_{1}, x_{2}, \ldots, x_{n}\right)$ in $D$ to the Diophantine equation. Of course, the Diophantine equation, or even the set $I_{i}$, could, for one or more values of $i$, preclude $\left|x_{i}\right|$ from becoming large. In a situation of this type the conditions (ii), (iii), and (iv) are vacuously satisfied for such values of $i$.

We now define the subclass $\Delta_{2}$ of $\Delta_{1}$ by replacing the set of $m$ limit conditions (iv) by a stronger set of conditions. The class $\Delta_{2}$ is defined to be the set of those Diophantine equations in $\Delta_{1}$ that are such that, for each $i \leqslant m$, there is a given real-valued function $\Omega=\Omega_{i}(w)$, of a real variable $w$, defined everywhere, that is continuous, that is strictly increasing as $w$ increases, whose inverse is given, and that possesses the following two properties. First, it is required that

(v) $\lim \Omega_{i}(w)=\infty$,

as $w$ approaches infinity. Secondly, it is required that

(vi) $\Omega_{i}\left(\left|x_{i}\right|\right) \leqslant \omega\left|q\left(x_{1}, x_{2}, \ldots, x_{n}\right)\right|$,

for all solutions $\left(x_{1}, x_{2}, \ldots, x_{n}\right)$ in $D$ to the Diophantine equation.

THEOREM 3. For each Diophantine equation in the class $\Delta_{2}$, the bound $\left|x_{i}\right| \leqslant B(k)$, for $1 \leqslant i \leqslant m$, is computable.

Proofs. Let us assume that there is such a function $q$ that satisfies (i), (ii), and (iii). Then there is a constant $a$ so that, by (i) and (ii), for each $i \leqslant m$, and for all solutions $\left(x_{1}, x_{2}, \ldots, x_{n}\right)$ in $D$ to the Diophantine equation, one has

$$
0 \leqslant \frac{f\left(x_{1}, x_{2}, \ldots, x_{n}\right)}{q\left(x_{1}, x_{2}, \ldots, x_{n}\right)}=\frac{|k|}{\left|q\left(x_{1}, x_{2}, \ldots, x_{n}\right)\right|}<1,
$$

if $\left|x_{i}\right|>a$. For such values of $x_{i}$ we then have

$$
\omega\left|q\left(x_{1}, x_{2}, \ldots, x_{n}\right)\right|=|k|,
$$

and so, by (iii), $\left|x_{i}\right| \leqslant x_{0}$. The Diophantine equation is realized to be in the class $\Delta$.

Now, if the set of conditions (i), (ii), and (iii) were to fail to hold, then for any choice of $q$ satisfying (i) and (ii), one would have, for any given constant $c>0$, a solution $\left(x_{1}, x_{2}, \ldots, x_{n}\right)$ in $D$ to the Diophantine equation, and a value of $i \leqslant m$, for which $\omega\left|q\left(x_{1}, x_{2}, \ldots, x_{n}\right)\right|=|k|$ and $\left|x_{i}\right|>c$, so that the Diophantine equation could not be in $\Delta$.

Let us now assume that there is such a function $q$ that satisfies (i) and (iv). Then, since $\omega$ can be given, for all $\left(x_{1}, x_{2}, \ldots, x_{n}\right)$ in $D$, by

$$
\omega=\frac{f\left(x_{1}, x_{2}, \ldots, x_{n}\right)}{q\left(x_{1}, x_{2}, \ldots, x_{n}\right)}-\left[\frac{f\left(x_{1}, x_{2}, \ldots, x_{n}\right)}{q\left(x_{1}, x_{2}, \ldots, x_{n}\right)}\right],
$$


we infer, if $x_{1}, x_{2}, \ldots, x_{n}$ satisfy the Diophantine equation, that $\omega q\left(x_{1}, x_{2}, \ldots, x_{n}\right)$ $\leqslant k$ if $k>0$ and that $\omega q\left(x_{1}, x_{2}, \ldots, x_{n}\right) \geqslant k$ if $k<0$, since, by (i), $k$ and $q$ have the same sign. Consequently, in either case one has an inequality, $\omega\left|q\left(x_{1}, x_{2}, \ldots, x_{n}\right)\right|$ $\leqslant|k|$, which also holds if $k=0$. It now follows from our limit hypotheses (iv) that there are only finitely many solutions $x_{1}, x_{2}, \ldots, x_{m}$ for all choices for $x_{m+1}, x_{m+2}, \ldots, x_{n}$ and for all choices of $k$ in any interval of finite length, so that the Diophantine equation is in $\Delta_{1}$.

Now, let it be assumed that the Diophantine equation is in $\Delta_{1}$. Then, for any given positive constant $c$, the Diophantine inequality $\left|f\left(x_{1}, x_{2}, \ldots, x_{n}\right)\right|<c$ has only finitely many solutions $x_{1}, x_{2}, \ldots, x_{m}$ for all values of $x_{m+1}, x_{m+2}, \ldots, x_{n}$. Let $x_{0}$ be the maximum of the absolute values of these solutions, if there are any, and let $x_{0}=0$, otherwise. Then it is the case that $\left|f\left(x_{1}, x_{2}, \ldots, x_{n}\right)\right| \geqslant c$ if, for any $i \leqslant m$, $\left|x_{i}\right|>x_{0}$. Let $q$ be defined by $q\left(x_{1}, x_{2}, \ldots, x_{n}\right)=2 \operatorname{sign}(k)\left|f\left(x_{1}, x_{2}, \ldots, x_{n}\right)\right|$, where $\operatorname{sign}(k)=1$ if $k \geqslant 0$ and $\operatorname{sign}(k)=-1$ if $k<0$, if $\left(x_{1}, x_{2}, \ldots, x_{n}\right)$ in $D$ is such that $f\left(x_{1}, x_{2}, \ldots, x_{n}\right) \neq 0$, and let $q\left(x_{1}, x_{2}, \ldots, x_{n}\right)=\operatorname{sign}(k)$ for the possible finite number of remaining values for $\left(x_{1}, x_{2}, \ldots, x_{n}\right)$ in $D$. We observe first that $k q\left(x_{1}, x_{2}, \ldots, x_{n}\right)>0$ if $k \neq 0$, for all choices of $\left(x_{1}, x_{2}, \ldots, x_{n}\right)$ in $D$, and then that $\omega\left|q\left(x_{1}, x_{2}, \ldots, x_{n}\right)\right|=\left|f\left(x_{1}, x_{2}, \ldots, x_{n}\right)\right|$ approaches infinity, uniformly in $x_{1}$, $x_{2}, \ldots, x_{i-1}, x_{i+1}, \ldots, x_{n}$, as $\left|x_{i}\right|$ approaches infinity, for each $i \leqslant m$, so that (i) and (iv) hold.

Suppose now that there is such a function $\Omega$ with the properties (v) and (vi). Then, one has $\Omega_{i}\left(\left|x_{i}\right|\right) \leqslant \omega\left|q\left(x_{1}, x_{2}, \ldots, x_{n}\right)\right| \leqslant|k|$, if $\left(x_{1}, x_{2}, \ldots, x_{n}\right)$ in $D$ satisfies the Diophantine equation, and if $i \leqslant m$. That is to say, all solutions $x_{i}$ in $I_{i}$ of our Diophantine equation lie among the solutions to the Diophantine inequality $\Omega_{i}\left(\left|x_{i}\right|\right)$ $\leqslant|k|$. If $|k|$ were to fail to be in the range of $\Omega$, then, since $\Omega$ is continuous and approaches infinity, it would be the case that $\Omega_{i}\left(\left|x_{i}\right|\right)>|k|$. It is then realized that $|k|$ is in the range of $\Omega$, and, hence, in the domain of the inverse function $\Omega^{-1}$. It now follows from our Diophantine inequality, since $\Omega$ is strictly increasing, that $\left|x_{i}\right| \leqslant \Omega_{i}^{-1}(|k|)$. Indeed, if one had $\left|x_{i}\right|>\Omega_{i}^{-1}(|k|)$, then one would have

$$
\Omega_{i}\left(\left|x_{i}\right|\right)>\Omega_{i}\left(\Omega_{i}^{-1}(|k|)\right)=|k| .
$$

We now focus attention back on the Diophantine equation to draw the conclusion that, for all $i$ with $1 \leqslant i \leqslant m$, it is the case that $\left|x_{i}\right| \leqslant B(k)$, where

$$
B(k)=\max \left(\Omega_{1}^{-1}(|k|), \Omega_{2}^{-1}(|k|), \ldots, \Omega_{m}^{-1}(|k|)\right) .
$$

Remark. The initial problem is usually to solve a Diophantine equation $f\left(x_{1}, x_{2}, \ldots, x_{n}\right)=k$ as $x_{1}, x_{2}, \ldots, x_{n}$ vary over all integers. On occasion, the construction of our function $\Omega$ depends upon the variables being positive. Since our bound will certainly hold for $x_{i}=0$, we can replace $x_{i}$ in the original Diophantine equation by $-x_{i}$, to reduce the problem to that of computing a bound for a positive integer variable $x_{i}$ for two Diophantine equations. Continuing in this manner, we see that the original problem is equivalent to that of computing a bound for a set $2^{n}$ Diophantine equations in positive integer variables. These derived Diophantine equations often satisfy the hypotheses of our algorithm if the original one does for positive values of the variables. In this situation, we let each $I_{i}$ be the set of all 
positive integers and compute a bound for this set of $2^{n}$ equations and, hence, a bound for the original Diophantine equation $f\left(x_{1}, x_{2}, \ldots, x_{n}\right)=k$ for all integer values of $x_{1}, x_{2}, \ldots, x_{n}$.

On other occasions the construction of $\Omega$ depends on each variable being in the range $\left|x_{i}\right| \geqslant x_{0}$ for a given constant $x_{0}$. In this situation, we would let each $I_{i}$ be the set of all integers with absolute value at least as large as $x_{0}$. We then apply the methods of our algorithm to compute a bound for $f\left(x_{1}, x_{2}, \ldots, x_{n}\right)=k$ as $\left(x_{1}, x_{2}, \ldots, x_{n}\right)$ varies over $D$. Then, by letting

$$
B(k)=\max \left(\Omega_{1}^{-1}(|k|), \Omega_{2}^{-1}(|k|), \ldots, \Omega_{n}^{-1}(|k|), x_{0}\right),
$$

we obtain a bound $\left|x_{i}\right| \leqslant B(k)$ for all integer solutions $x_{1}, x_{2}, \ldots, x_{n}$ to $f\left(x_{1}, x_{2}, \ldots, x_{n}\right)=k$.

3. A Cubic Polynomial Diophantine Equation. As a first illustration of the ideas, let us solve the equation

$$
x y^{2}+y+k=2 x^{3} \text {. }
$$

We assert that if $k=0$, then the solutions are: $x=0, y=0 ; x= \pm 1, y= \pm 1$; and $x= \pm 1, y=\mp 2$. If $k \neq 0$, then all solutions satisfy

$$
|x|<10|k| \text {. }
$$

In particular, the Diophantine equation (1) has only finitely many solutions for a specified integer $k$.

The case $k=0$ can be dispensed with at once. If $x \neq 0$, then $x$ divides $y$ so that $y=x z$. Thus we have $x^{3} z^{2}+x z=2 x^{3}$, so that $x^{2}$ divides $z$. We then have $z=w x^{2}$ and $w^{2} x^{7}+w x^{3}=2 x^{3}$, so that $w$ divides 2. That is to say, $y$ is given by $y= \pm x^{3}$, $\pm 2 x^{3}$. In the first case we observe that $x^{7} \pm x^{3}=2 x^{3}$, and hence that $x= \pm 1$ and $y= \pm 1$. In the second case one obtains $4 x^{7} \pm 2 x^{3}=2 x^{3}$ from which we infer that $x= \pm 1$ and $y=\mp 2$.

In order to simplify the proof we shall reduce the problem of solving the equation (1) with $k \neq 0$ to that of solving an equation with $x$ and $y$ both positive.

If $x=0$, then the bound (2) certainly holds. If $y=0$, then the equation (1) has a solution only if $k$ is twice a cube and then $x=\sqrt[3]{k / 2}$ so that the bound (2) is realized. If $x<0$ and $y>0$, then the equation (1) is equivalent to $x y^{2}-y-k=2 x^{3}$ with $x, y>0$. If $x>0$ and $y<0$, then our equation (1) is equivalent to $x y^{2}-y+k$ $=2 x^{3}$ with $x, y>0$. If $x<0$ and $y<0$, then the equation (1) is equivalent to $x y^{2}+y-k=2 x^{3}$ where $x, y>0$.

It is then realized that our original Diophantine equation (1) with $k \neq 0$ is equivalent to

$$
x y^{2}+a y+k=2 x^{3}
$$

where $k \neq 0, a= \pm 1$ and $x, y>0$. We shall deduce the bound (2) for the equation (3).

4. The Case $k>0$. From the equation (3) we get that

$$
y=-\frac{a}{2 x} \pm \sqrt{\frac{1}{4 x^{2}}-\frac{k}{x}+2 x^{2}} .
$$


If the minus sign did occur, then one would have

$$
-\frac{a}{2 x}>\sqrt{\frac{1}{4 x^{2}}-\frac{k}{x}+2 x^{2}},
$$

and so $2 x^{3}<k$. Hence $y$ is given by

$$
y=-\frac{a}{2 x}+\sqrt{\frac{1}{4 x^{2}}-\frac{k}{x}+2 x^{2}},
$$

if $x$ satisfies

$$
x \geqslant \sqrt[3]{\frac{k}{2}}
$$

Since

$$
\sqrt{\frac{1}{4 x^{2}}-\frac{k}{x}+2 x^{2}}<\sqrt{2} x+1+\frac{a}{2 x},
$$

it follows that the inequality $y<\sqrt{2} x+1$ always holds. Also we have $y>\sqrt{2} x-1$ if the inequality

$$
\sqrt{\frac{1}{4 x^{2}}-\frac{k}{x}+2 x^{2}}>\sqrt{2} x-1+\frac{a}{2 x}
$$

is realized. If the right-hand side is positive, that is, if $x \geqslant 2$ then, by squaring, we get that this inequality is equivalent to

$$
2 \sqrt{2} x^{2}-(1+\sqrt{2} a) x+a>k \text {. }
$$

If $a=-1$, this inequality holds if $x \geqslant 3$ and $x>\sqrt{k / 2 \sqrt{2}}$. If $a=1$, it holds if $x>\sqrt{k / \sqrt{2}}$. We have shown that $y>\sqrt{2} x-1$ if $x$ satisfies $x \geqslant 3$ and

$$
x>\sqrt{\frac{k}{\sqrt{2}}} .
$$

Let $q(x, y)=x$. Then $\omega$ is given by

$$
\omega=\left\{\frac{2 x^{3}-x y^{2}-a y}{x}\right\}=\left\{\frac{-a y}{x}\right\} .
$$

If $a=1$, then $\omega=\{-\sqrt{2}+\varepsilon\}$, where $|\varepsilon|<1 / x$. Thus $\omega=2-\sqrt{2}+\varepsilon$, if $\varepsilon$ satisfies $0 \leqslant 2-\sqrt{2}+\varepsilon<1$. Let us require a stronger condition on $\varepsilon$, namely that

$$
0.1<2-\sqrt{2}+\varepsilon<1 \text {. }
$$

Then we have $\omega$ bounded away from zero: $\omega>0.1$. This restriction on $\varepsilon$ will hold if $-0.48<\varepsilon<0.4$, that is, if $|\varepsilon|<0.4$, or, if $x \geqslant 3$.

If $a=-1$, then $\omega=\{\sqrt{2}+\varepsilon\}$, where $|\varepsilon|<1 / x$. Thus we infer that $\omega=$ $\sqrt{2}-1+\varepsilon$, if $\varepsilon$ satisfies $0 \leqslant \sqrt{2}-1+\varepsilon<1$. It will be the case that $\omega>0.1$ if $\varepsilon$ is required to satisfy the stronger condition that

$$
0.1<\sqrt{2}-1+\varepsilon<1 \text {. }
$$

This restriction will be satisfied if $|\varepsilon|<0.3$, or, if

$$
x \geqslant 4 \text {. }
$$


It is then concluded that $\omega>0.1$ if the inequalities (5), (6), and (7) all hold. Consequently, since

$$
\omega|q|=\left(\frac{|k|}{|q|}-\left[\frac{|k|}{|q|}\right]\right)|q| \leqslant|k|
$$

always holds, the bound

$$
x<10 k
$$

is realized. But, if any of the inequalities (5), (6), or (7) failed to hold, then the bound (8) certainly would be satisfied. We then draw the conclusion that the bound (8) holds for the equation (3) for all $x$ in the case that $k>0$.

5. The Case $k<0$. Let us now assume that $k<0$ in the equation (3). Then $y$ is given by (4), since the inequality (5) now automatically holds. This time it is concluded that $y<\sqrt{2} x+1$ if $x$ satisfies

$$
2 \sqrt{2} x^{2}+(1+\sqrt{2} a) x+a>|k| .
$$

This will occur if

$$
x>\sqrt{\frac{|k|}{\sqrt{2}}} .
$$

The inequality $y>\sqrt{2} x-1$ is now automatically satisfied.

This time we let $q(x, y)=-x$. Then one has

$$
\omega=\{a y / x\} .
$$

If $a=1$, then $\omega=\{\sqrt{2}+\varepsilon\}$ where $|\varepsilon|<1 / x$. Consequently, it is concluded that $\omega=\sqrt{2}-1+\varepsilon$ if $\varepsilon$ satisfies $0 \leqslant \sqrt{2}-1+\varepsilon<1$. We shall require $\varepsilon$ to satisfy the stronger condition that

$$
0.1<\sqrt{2}-1+\varepsilon<1,
$$

so that, then, $\omega>0.1$. Our condition on $\varepsilon$ is satisfied if $|\varepsilon|<0.3$, that is, if

$$
x \geqslant 4 \text {. }
$$

If $a=-1$, then $\omega\{-\sqrt{2}+\varepsilon\}$, where $|\varepsilon|<1 / x$. Let us assume that $0.1<$ $2-\sqrt{2}+\varepsilon<1$, so that $\omega=2-\sqrt{2}+\varepsilon$ and that $\omega>0.1$. This will happen if $|\varepsilon|<0.4$, or, if $x \geqslant 3$.

We have shown that the bound $x<10|k|$ holds for the equation (3) if the inequalities (9) and (10) are satisfied. If either of the inequalities (9) or (10) should fail to hold, then certainly this bound is obtained. Let it then be realized that all solutions to our original Diophantine equation (1) with $k \neq 0$ lie in the range $|x|<10|k|$.

6. A Quartic Polynomial Diophantine Equation. As a second illustration of the techniques, we shall study the equation

$$
\begin{aligned}
\left(a_{1} x^{2}+a_{2} x+a_{3}\right) y^{2}+\left(a_{4} x^{3}+a_{5} x^{2}\right. & \left.+a_{6} x+a_{7}\right) y \\
& +a_{8} x^{4}+a_{9} x^{3}+a_{10} x^{2}+a_{11} x=k .
\end{aligned}
$$


This furnishes an interesting illustration of our methods, because it is necessary to have specified conditions on the coefficients in order for a bound to exist, since there could be infinitely many solutions.

THEOREM 4. Let $a_{i}$, for $1 \leqslant i \leqslant 11$, and $k$ be given integers with $k$ not equal to zero. Assume that $a_{4}^{2}-4 a_{1} a_{8}$ is not equal to a square and that $a_{1}^{2} a_{6}-a_{1} a_{2} a_{5}-$ $a_{1} a_{3} a_{4}+a_{2}^{2} a_{4} \neq 0$. Then the Diophantine equation (11) has only finitely many solutions and an upper bound for $|x|$ can be computed. In fact, there is a bound of the form $|x|<\gamma \sqrt{|k|}$, where $\gamma$ is a computable constant that depends only on the $a_{i}$ 's.

7. Proof of Theorem 4. We begin by establishing the existence of a computable bound $|x| \leqslant B(k)$. If $x=0$, such a bound is certainly realized. If $x<0$, we can replace $x$ by $-x$ to reduce the problem to that of computing a bound for a positive integer variable $x$ for two Diophantine equations. The derived Diophantine equation satisfies the hypotheses of Theorem 4 if the original one does. This is due to the fact that $k$ and $a_{4}^{2}-4 a_{1} a_{8}$ are invariant under such a substitution; whereas, $a_{1}^{2} a_{6}-$ $a_{1} a_{2} a_{5}-a_{1} a_{3} a_{4}+a_{2}^{2} a_{4}$ is transformed into its negative. It is therefore sufficient to establish the theorem under the assumption that $x>0$.

By viewing $y$ as a function of a real variable $x$, given by Eq. (11), and by employing the binomial series, we realize that for all sufficiently large values of $x$, it is the case that $y$ has two expansions of the form

$$
y=\alpha_{0} x+\alpha_{1}+\frac{\alpha_{2}}{x}+\frac{\alpha_{3}}{x^{2}}+\ldots
$$

where $\alpha_{0}$ takes on the values

$$
\alpha_{0}=\frac{-a_{4} \pm \sqrt{a_{4}^{2}-4 a_{1} a_{8}}}{2 a_{1}}
$$

We shall assume that

$$
x \geqslant x_{0}
$$

where $x_{0}$ is a computable real constant, large enough so that the Laurent expansions (12) hold for all $x$ in the range of (14), and, at the same time, large enough so that all the conditions to follow also hold. We write the Laurent expansions (12) as

$$
y=\alpha_{0} x+\varepsilon_{1},
$$

where

$$
\varepsilon_{1}=\alpha_{1}+\frac{\alpha_{2}}{x}+\frac{\alpha_{3}}{x^{2}}+\ldots
$$

is estimated from

$$
\left|\varepsilon_{1}\right|<c_{1} \text {. }
$$

Here, $c_{1}$ and the $c_{i}$ 's to follow, all represent computable positive constants. If it is the case that $a_{4}^{2}-4 a_{1} a_{8}<0$, then by (13) and (16), we realize that there is no real, and hence no integral, value of $y$ given by (15), for $x$ in the range of (14). We thus assume that $a_{4}^{2}-4 a_{1} a_{8}>0$. 
We shall have occasion here to choose $q(x, y)$ in two ways, $q_{1}(x, y)$ and $q_{2}(x, y)$. Let $\omega_{1}$ and $\omega_{2}$ be, respectively, the corresponding values of $\omega$. We let $q_{1}(x, y)$ $=\operatorname{sign}(k) x^{2}$ and $q_{2}(x, y)=\operatorname{sign}(k) x^{3}$. Then, from the Diophantine equation (11), one has

$$
\begin{gathered}
\omega_{1}=\left\{\operatorname{sign}(k)\left(\frac{a_{2} y^{2}}{x}+\frac{a_{3} y^{2}}{x^{2}}+\frac{a_{6} y}{x}+\frac{a_{7} y}{x^{2}}+\frac{a_{11}}{x}\right)\right\}, \\
\omega_{2}=\left\{\operatorname { s i g n } ( k ) \left(\frac{a_{1} y^{2}}{x}+\frac{a_{2} y^{2}}{x^{2}}+\frac{a_{5} y}{x}+\frac{a_{3} y^{2}}{x^{3}}+\frac{a_{6} y}{x^{2}}\right.\right. \\
\left.\left.+\frac{a_{10}}{x}+\frac{a_{7} y}{x^{3}}+\frac{a_{11}}{x^{2}}\right)\right\} .
\end{gathered}
$$

The $y^{2} / x$ term in $\omega_{1}$ can be eliminated as follows. We write, by using (18),

$$
\begin{aligned}
\omega_{2}=\operatorname{sign}(k)\left(\frac{a_{1} y^{2}}{x}+\frac{a_{2} y^{2}}{x^{2}}+\frac{a_{5} y}{x}+\right. & \frac{a_{3} y^{2}}{x^{3}}+\frac{a_{6} y}{x^{2}} \\
& \left.+\frac{a_{10}}{x}+\frac{a_{7} y}{x^{3}}+\frac{a_{11}}{x^{2}}\right)-A,
\end{aligned}
$$

where $A$ is an integer given by

$$
\begin{aligned}
A=\left[\operatorname { s i g n } ( k ) \left(\frac{a_{1} y^{2}}{x}+\frac{a_{2} y^{2}}{x^{2}}+\frac{a_{5} y}{x}+\frac{a_{3} y^{2}}{x^{3}}\right.\right. & +\frac{a_{6} y}{x^{2}} \\
& \left.\left.+\frac{a_{10}}{x}+\frac{a_{7} y}{x^{3}}+\frac{a_{11}}{x^{2}}\right)\right] .
\end{aligned}
$$

We first substitute the value of the quantity $y^{2} / x$, obtained from formula (19), into the expression (17) for $\omega_{1}$, and then we eliminate $y$ in the resulting $\omega_{1}$, by using (15), to compute that $\omega_{1}$ has the form

$$
\omega_{1}=\left\{\operatorname{sign}(k)\left(\left(a_{3}-\frac{a_{2}^{2}}{a_{1}}\right) \alpha_{0}^{2}+\left(a_{6}-\frac{a_{2} a_{5}}{a_{1}}\right) \alpha_{0}\right)+\left\{\frac{a_{2} A}{a_{1}}\right\}+\varepsilon_{2}\right\}
$$

Here, $\varepsilon_{2}$ is a quantity of the type

$$
\varepsilon_{2}=\frac{a_{2} \omega_{2}}{a_{1}}+\varepsilon_{3}
$$

where $\varepsilon_{3}$ is a quantity that, in view of (16), can be estimated by

$$
\left|\varepsilon_{3}\right|<c_{2} / x \text {. }
$$

Let us assume that

$$
\omega_{2}<1 / x .
$$

Then, from (22), (23), and (21), one has an estimation

$$
\left|\varepsilon_{2}\right|<c_{3} / x \text {. }
$$

Let us now define a function $\Omega_{0}$ of $x$ as follows:

$$
\Omega_{0}=\left\{\operatorname{sign}(k)\left(\left(a_{3}-\frac{a_{2}^{2}}{a_{1}}\right) \alpha_{0}^{2}+\left(a_{6}-\frac{a_{2} a_{5}}{a_{1}}\right) \alpha_{0}\right)+\left\{\frac{a_{2} A}{a_{1}}\right\}\right\} .
$$


Then, from (20) and (25), $\omega_{1}$ is evaluated as

$$
\omega_{1}=\Omega_{0}+\varepsilon_{2},
$$

provided $\varepsilon_{2}$ lies in the range given by $0 \leqslant \Omega_{0}+\varepsilon_{2}<1$. We shall require the stronger inequality

$$
-\Omega_{0} / 2<\varepsilon_{2}<1-\Omega_{0}
$$

to be valid. This will occur if

$$
\left|\varepsilon_{2}\right|<\min \left(\frac{\Omega_{0}}{2}, 1-\Omega_{0}\right) .
$$

It follows from (26) and (27) that $\omega_{1}$ has a lower bound given by

$$
\omega_{1}>\Omega_{0} / 2 \text {. }
$$

We shall argue that $\Omega_{0}$, as a function of an integer variable $x$, has a positive minimum. First, we observe, in view of (13), that

$$
\begin{aligned}
\left(a_{3}-\right. & \left.\frac{a_{2}^{2}}{a_{1}}\right) \alpha_{0}^{2}+\left(a_{6}-\frac{a_{2} a_{5}}{a_{1}}\right) \alpha_{0} \\
= & \pm \frac{1}{2 a_{1}^{3}}\left(a_{1}^{2} a_{6}-a_{1} a_{2} a_{5}-a_{1} a_{3} a_{4}+a_{2}^{2} a_{4}\right) \sqrt{a_{4}^{2}-4 a_{1} a_{8}} \\
& +\frac{1}{2 a_{1}^{3}}\left(-2 a_{1}^{2} a_{3} a_{8}-a_{1}^{2} a_{4} a_{6}+2 a_{1} a_{2}^{2} a_{8}+a_{1} a_{2} a_{4} a_{5}+a_{1} a_{3} a_{4}^{2}-a_{2}^{2} a_{4}^{2}\right) .
\end{aligned}
$$

Secondly, we note that the quantity (30) is irrational. Now, since the function $\left\{a_{2} A / a_{1}\right\}$ only takes on the possible values

$$
\left\{\frac{a_{2} A}{a_{1}}\right\}=0, \frac{1}{a_{1}}, \frac{2}{a_{1}}, \ldots, \frac{a_{1}-1}{a_{1}},
$$

it follows from our definition (25) of $\Omega_{0}$ that

$$
\min \Omega_{0}>0 \text {. }
$$

The same argument shows that

$$
\min \left(1-\Omega_{0}\right)>0 \text {. }
$$

In order to have the desired lower bound (29) for $\omega_{1}$, the inequality (28) must be realized. But from (31), (32), and (24), we infer that the inequality (28) will be valid if

$$
\frac{c_{3}}{x}<\min \left(\min \frac{\Omega_{0}}{2}, \min \left(1-\Omega_{0}\right)\right) .
$$

It is then realized that for $x$ in the range of (14), the inequality (28) will occur.

In the notation of our algorithm, we let $I_{1}$ be the set of all integers that are at least as large as $x_{0}$; we let $I_{2}$ be the set of all integers; we let $m=1$; and we define $q(x, y)$ and $\Omega$ as follows. If $\omega_{2} \geqslant 1 / x$, let $q(x, y)=q_{2}(x, y)=\operatorname{sign}(k) x^{3}$ and let $\Omega_{1}(x)=x^{2}$. Otherwise, the inequality (23) holds and we then define $q(x, y)=$ $q_{1}(x, y)=\operatorname{sign}(k) x^{2}$ and $\Omega_{1}(x)=\left(\min \Omega_{0}\right) x^{2} / 2$. If $x$ is in $I_{1}$, so that the inequality (14) holds, then, in view of (29) and (31), we see that all the hypotheses of our algorithm are satisfied. It is then realized that there is a computable bound for the Diophantine equation (11), for all values of $x$ in $I_{1}$. By taking the larger of this 
bound and $x_{0}$, we realize that there is a computable bound for all positive integer solutions $x$, and hence, as noted earlier, there is a computable bound $|x| \leqslant B(k)$ for all integer solutions $x$ to the Diophantine equation (11), subject to the restrictions of Theorem 4.

The essential idea in modifying the above proof to one that yields a bound of the form $|x|<\gamma \sqrt{|k|}$ is to make the observation that if it is assumed that $x>\sqrt[3]{|k|}$, then the quantities $x_{0}$ and $c_{1}$ can be computed to be independent of $k$. The details of the calculation of $\gamma$ are left to the reader.

8. The Class $\Delta_{1}$. In order to acquire some insight into the nature of the class $\Delta_{1}$ we shall establish the following.

Proposition. The class $\Delta_{1}$ includes as a subclass the class of all Diophantine equations included in Thue's theorem.

Proof. We are considering Diophantine equations of the type $f(x, y)=k$, with $k$ a given nonzero integer and

$$
f(x, y)=a_{0} x^{n}+a_{1} x^{n-1} y+a_{2} x^{n-2} y^{2}+\cdots+a_{n} y^{n}
$$

an irreducible binary form with given integer coefficients and with a given degree $n \geqslant 3$. In the notation of our algorithm, we let $I_{i}$, for $i=1,2$, be the set of all integers, and we choose $m$ in the optimal manner, $m=2$. One can write

$$
f(x, y)=a_{n} x^{n} \prod_{i=1}^{n}\left(\frac{y}{x}-\alpha_{i}\right),
$$

where $\alpha_{1}, \alpha_{2}, \ldots, \alpha_{n}$ are the roots of the irreducible equation

$$
a_{n} z^{n}+a_{n-1} z^{n-1}+\cdots+a_{0}=0,
$$

and where $a_{n} \neq 0$. Let $\gamma=\min \left|\alpha_{i}-\alpha_{j}\right|$, where the minimum is taken under all $i$ and $j$ with $i \neq j$. Then, it follows from the irreducibility condition that $\gamma>0$. If it were the case that, for all $i,\left|y / x-\alpha_{i}\right| \geqslant \gamma / 2$, then it would be the case that

$$
|f(x, y)| \geqslant\left|a_{n}\right|\left(\frac{\gamma}{2}\right)^{n}|x|^{n}>|k|
$$

if

$$
|x|>\frac{2}{\gamma} \sqrt[n]{\frac{|k|}{\left|a_{n}\right|}} .
$$

This would contradict the Diophantine equation. It is then the case that one of the $\alpha_{i}$ 's, which we assume to be $\alpha_{1}$, has the property that $\left|y / x-\alpha_{1}\right|<\gamma / 2$ for all values of $x$ in the above range. We let $j \neq 1$ and write

$$
\gamma \leqslant\left|\alpha_{j}-\alpha_{1}\right|=\left|\left(\frac{y}{x}-\alpha_{1}\right)-\left(\frac{y}{x}-\alpha_{j}\right)\right| \leqslant\left|\frac{y}{x}-\alpha_{1}\right|+\left|\frac{y}{x}-\alpha_{j}\right| .
$$

It is then realized that for all $j \neq 1,\left|y / x-\alpha_{j}\right|>\gamma / 2$. We define $q(x, y)=\operatorname{sign}(k)$ if $x=0$ and $q(x, y)=\operatorname{sign}(k)|x|^{n}$ if $x \neq 0$. It is then realized that $k q(x, y)>0$ for all integers $x$ and $y$. It is also realized that $\omega=\left\{|k| /|x|^{n}\right\}$ if $x \neq 0$. Let us 
assume that $|x|>\sqrt[n]{|k|}$. Then, $\omega$ is given by

$$
\omega=\frac{|k|}{|x|^{n}}=\left|a_{n}\right|\left|\frac{y}{x}-\alpha_{1}\right| \prod_{i=2}^{n}\left|\frac{y}{x}-\alpha_{i}\right| .
$$

If we further assume that $|x|>2 \sqrt[n]{|k| /\left|a_{n}\right|} / \gamma$, then we have

$$
\omega>\left|a_{n}\right|\left|\frac{y}{x}-\alpha_{1}\right|\left(\frac{\gamma}{2}\right)^{n-1} .
$$

Now, let $\varepsilon>0$. Then, by the Thue-Siegel-Roth Theorem, there is a positive constant $c=c(\varepsilon)$ so that one has

$$
\left|\frac{y}{x}-\alpha_{1}\right|>\frac{c}{|x|^{2+\varepsilon}}
$$

since $\alpha_{1}$ is irrational. It is now concluded that

$$
\omega|q(x, y)|>c\left|a_{n}\right|\left(\frac{\gamma}{2}\right)^{n-1}|x|^{n-2-\varepsilon}
$$

and hence that $\lim \omega|q(x, y)|=\infty$ as $|x|$, or equivalently, $|y|$, approaches infinity, if we choose $\varepsilon<1$.

It should be noted that the constant $c$ of the Thue-Siegel-Roth Theorem is still, at this date, incomputable.

The reader may be interested in showing that the nonpolynomial Diophantine equation $x y^{2} z^{3}+y+x z^{2}[\log |x|]+k=2 x^{3} z^{5}$ in three variables $x, y$, $z$, with $x, z \neq 0$, is in the class $\Delta_{1}$, and that an upper bound for $|x|,|y|$, and $\mid z \uparrow$ can be computed, in analogy with the cubic equation of Section 3.

9. Concluding Remarks. The quartic equation of Section 6 furnishes us with a good first illustration of my methods, because the choices of $q(x, y)$, the computation of the Laurent series for $y$, and the analysis of $\omega$, are all relatively simple.

In a more realistic example, the function $q(x, y)$ is chosen in a variety of ways, according to a number of different cases, the expansions for $y$ would be those of Puiseux series, and the analysis of $\omega$ would be more intricate. This analysis would involve not only the constant term in $\omega$, but also terms such as those of the form $1 / x, 1 / x^{2}$, and so on. To make matters even worse, there could occur such unwieldy terms as $x, x^{2}$, and so on, multiplied by irrational coefficients, or terms such as $\sqrt{x}$. These terms must be eliminated. This is done by a process that I shall call analytic elimination, which involves manipulation of the Puiseux series expansions of $y$. We were able to avoid this phenomenon in our illustration of the quartic equation of Section 6 , by eliminating, at the outset, the term $y^{2} / x$.

As I mentioned earlier, I have extended the methods introduced in Part I of this paper and I have applied these resulting methods to the general quartic equation, under certain hypotheses. These hypotheses can be conveniently formulated in terms of a quantity that we shall call the leading part of a polynomial. Let

$$
F(x, y)=\sum_{i=0}^{d_{1}} \sum_{j=0}^{d_{2}} a_{i j} x^{i} y^{j}
$$


be a polynomial in $x$ and $y$, of degree $d_{1}$ and $d_{2}$ in $x$ and $y$, respectively. Let $\lambda$ be any positive real number. We define the $\lambda$-leading part of $F(x, y)$, denoted by $F_{\lambda}(x, y)$, to be the polynomial consisting of the sum of all nonzero terms $a_{i j} x^{i} y^{j}$ of $F(x, y)$ for which $i+\lambda j$ is maximal, for that fixed value of $\lambda$. We define the leading part of $F(x, y)$, denoted by $\tilde{F}(x, y)$, to be the polynomial consisting of the sum of all such terms, as $\lambda$ varies. A related notion is that of the leading form of $F(x, y)$, which is the polynomial consisting of the sum of all terms of $F(x, y)$ of maximal degree. It is, in the present notation, $F_{1}(x, y)$.

For example, if $F(x, y)=y^{4}+y^{3}-2 x^{2} y^{2}+x y+3 x^{3}+x-5$, then $F_{\lambda}(x, y)$ $=y^{4}, y^{4}-2 x^{2} y^{2},-2 x^{2} y^{2},-2 x^{2} y^{2}+3 x^{3}$, or $3 x^{3}$, according as $\lambda>1, \lambda=1$, $\frac{1}{2}<\lambda<1, \lambda=\frac{1}{2}$, or $\lambda<\frac{1}{2}$, respectively. Thus, $\tilde{F}(x, y)=y^{4}-2 x^{2} y^{2}+3 x^{3}$. If $F(x, y)=y^{4}+x y^{2}-2 x^{3}-18$, then $F_{\lambda}(x, y)=y^{4}, y^{4}-2 x^{3}$, or $-2 x^{3}$, according as $\lambda>\frac{3}{4}, \lambda=\frac{3}{4}$, or $\lambda<\frac{3}{4}$, respectively. In this case $\tilde{F}(x, y)=F_{3 / 4}(x, y)=$ $y^{4}-2 x^{3}$.

The result for the general quartic equation, which is established by employing my methods, in Part II of this paper (Hilliker [3]), can be formulated as follows: If the general quartic polynomial Diophantine equation, in two variables, with integer coefficients, is irreducible, and if, in addition, either the leading part is not a constant multiple of a power of an irreducible polynomial, or, the leading part is not equal to any $\lambda_{0}$-leading part, then there are only finitely many solutions and an upper bound for the absolute value of each solution can be computed.

By the leading part of a polynomial equation we mean, after it is put into the form $F(x, y)=0$, the leading part of the polynomial $F(x, y)$. When we say that a polynomial equation, with integer coefficients, is irreducible, we mean, after it is put into the form $F(x, y)=0$, that $F(x, y)$ is irreducible. That is to say, we mean that $F(x, y)$ cannot be expressed as the product of two nonconstant polynomials with rational coefficients.

For example, let us consider the Diophantine equation

$$
y^{4}+y^{3}-2 x^{2} y^{2}+x y+3 x^{3}+x-5=0 .
$$

We note that this equation is irreducible. Indeed, any possible factorization would result from extending the factorization $y^{2}\left(y^{2}-2 x^{2}\right)$ of the leading form:

$$
\begin{aligned}
y^{4}+y^{3}-2 x^{2} y^{2}+ & x y+3 x^{3}+x-5 \\
& =\left(y^{2}+a_{1} x+a_{2} y+a_{3}\right)\left(y^{2}-2 x^{2}+a_{4} x+a_{5} y+a_{6}\right) .
\end{aligned}
$$

If we equate the coefficients on both sides of this equation, we obtain a system of equations in the $a_{i}$ 's that has no solution. This Diophantine equation is then covered by the hypotheses of the above theorem, and hence it has only finitely many solutions. An example of an irreducible Diophantine equation that is not covered by these hypotheses would be $y^{4}+x y^{2}-2 x^{3}-18=0$.

We now formulate a version of Runge's result for an equation of an arbitrary degree that is equivalent to that of his paper of 1887 , in exact analogy with the above theorem for the general quartic equation. Let $F(x, y)$ be a polynomial, in two variables $x$ and $y$, with integer coefficients, that is irreducible. We say that such a polynomial satisfies Runge's Condition unless, for some $\lambda_{0}, \tilde{F}(x, y)=F_{\lambda_{0}}(x, y)$ is a constant multiple of a power of an irreducible polynomial. Runge's Theorem is 
stated as follows: If $F(x, y)$ satisfies Runge's Condition, then the Diophantine equation $F(x, y)=0$ has only finitely many solutions and an upper bound for $|x|$ and $|y|$ can be computed.

Runge did not compute a bound for $|x|$ and $|y|$ in his paper of 1887 . E. G. Straus and I (Hilliker and Straus [6]) have made such a computation. Let $h$ be the height of $F(x, y)$, that is to say, the maximum of the absolute values of the coefficients of $F(x, y)$. Let $d$ be the maximum of the degrees $d_{1}$ and $d_{2}$ in $x$ and $y$, respectively. Then the following bound, involving only $h$ and $d$, is realized, for those Diophantine equations that satisfy Runge's Condition:

$$
\max (|x|,|y|)<(8 d h)^{d^{2 d^{3}}} .
$$

A special case of Runge's Theorem appears in Mordell's book [11] where stronger hypotheses are stated (Chapter 28, Theorem 1).

We close Part I of this paper by making the observation that what we gave to our algorithm in Section 2 was a proof. It is interesting to wonder if one can somehow systematically derive such algorithms. Specifically, I pose the following problem: To state a master algorithm for systematically deriving classes of Diophantine equations, and for systematically deriving for each such class an algorithm for solving each Diophantine equation in that particular class. Briefly, I pose the problem in these words: To state an algorithm for deriving algorithms for solving Diophantine equations.

Department of Computer Science

California State University

Fullerton, California 92634

Department of Mathematics

University of California

Los Angeles, California 90024

1. Alan Baker, Transcendental Number Theory, Cambridge Univ. Press, London and New York, 1975. See MR 54 \# 10163.

2. Ove Hemer, On the Diophantine Equation $y^{2}-k=x^{3}$, Doctoral Dissertation, Almqvist \& Wiksells, Uppsala, 1952. See MR 14, p. 354; Reviews in Number Theory, D24-24.

3. DAvid LeE Hilliker, "An algorithm for solving a certain class of Diophantine equations. II,"(to be submitted).

4. David Lee Hilliker \& Ray Steiner, "On Mordell's Diophantine equation," (to be submitted).

5. David Lee Hilliker \& E. G. Straus, "On Puiseux series whose curves pass through an infinity of algebraic lattice points," (to be submitted).

6. David Lee Hilliker \& E. G. Straus, "Determination of bounds for the solutions to those binary Diophantine equations that satisfy the hypotheses of Runge's theorem," (to be submitted).

7. Hymie London \& Raphael Finkelstein, On Mordell's Equation $y^{2}-k=x^{3}$, Bowling Green State Univ. Press, Bowling Green, Ohio, 1973. See MR 49 \#4928.

8. EDMOND MAILlET, "Sur les équations indéterminées à deux et trois variables qui n'ont qu'un nombre fini de solutions en nombres entieres," J. Math. Pures Appl., v. 6 (5), 1900, pp. 261-277. See Jbuch., Vol. 30, pp. 188-189.

9. EDMOND MAILLET, "Sur une catégorie d'équations indéterminées n'ayant en nombres entiers qu'un nombre fini de solutions," Nouv. Ann. de Math., v. 18, Series 4, 1918, pp. 281-292. See Jbuch., Vol. 31, pp. 190-191.

10. Louis Joel MoRdel, $A$ Chapter in the Theory of Numbers, An Inaugural Lecture, Cambridge Univ. Press, London, 1947. See Zbl., Vol. 31, p. 108. A related work of Mordell appeared: Three Lectures on Fermat's Last Theorem, Cambridge Univ. Press, London, 1921. See Jbuch., Vol. 48, pp. 129, 1164. This work of 1921 was reprinted by Chelsea, New York, 1955, as part of the book, Famous Problems and Other Monographs, by F. Klein, et al. See also: Le Dernier Théorème de Fermat, Les Presses Universitaires de France, Paris, 1929. See Jbuch., Vol. 55, p. 694. Mordell's two booklets of 1921 and 1947 were reprinted as one volume: Two Papers on Number Theory. It has an Introduction by O. Neumann, VEB Deutscher Verlag der Wissenschaften, Berlin, 1972. 
11. Louis Joel Mordell, Diophantine Equations, Academic Press, London and New York, 1969. See MR 40 \# 2600; Reviews in Number Theory, D02-23.

12. Trygve Nagell, "Einige Gleichungen von der Form $a y^{2}+b y+c=d x^{3}$," Avh. Norske Vid.-Akad. Oslo, Mat.-Natur. Kl., No. 7, 1930, 15 pp. See Jbuch., Vol. 56, p. 877.

13. C. RUNGE, "Ueber ganzzahlige Lösungen von Gleichungen zwischen zwei Veränderlichen," J. Reine Angew. Math., v. 100. 1887, pp. 425-435. See Jbuch., Vol. 19, pp. 76-77.

14. A. SCHInzel, "An improvement of Runge's theorem on Diophantine equations," Comment. Pontificia Acad. Sci., v. 2, no. 20, 1969, pp. 1-9. See MR 43 \# 1922.

15. TH. SKolem, "Über ganzzahlige Lösungen einer Klasse unbestimmter Gleichungen," Norsk Mat. Forenings Skrifter, Serie I, Nr. 10, 1922, 12 pp. See Jbuch., Vol. 48, p. 139.

16. TH. Skolem, Diophantische Gleichungen, Verlag von Julius Springer, Berlin, 1938; reprinted by Chelsea, New York, 1950. See Jbuch., Vol. 64, p. 112. 